\title{
La didáctica de la alineación de los procesos de producción en planta y la información a través de la herramienta MESView
}

\section{Pedro Gómez-Gasquet ${ }^{a}$, Manuel Díaz-Madroñero ${ }^{b}$ y Francisco Fraile ${ }^{c}$}

${ }^{a}$ Universitat Politècnica de Valencia, Departamento de Organización de Empresas, Centro de Investigación Gestión e Ingeniería de Producción (CIGIP) (pgomez@cigip.upv.es )

${ }^{\mathrm{b}}$ Universitat Politècnica de Valencia, Departamento de Organización de Empresas, Centro de Investigación Gestión e Ingeniería de Producción (CIGIP) (fcodiama@ cigip.upv.es)

${ }^{\mathrm{c}}$ Universitat Politècnica de Valencia, Instituto Universitario de Telecomunicación y Aplicaciones Multimedia (i-TEAM) (ffraile@iteam.upv.es)

\begin{abstract}
This paper presents the experience in using a manufacturing execution system, called MESView, for practical sessions of the course in Information Systems belonging to Master's Degree in Organisational and Logistics Engineering at UPV. Through a simple example based on a company belonging to food industrial sector, different system utilities of the selected manufacturing execution system, its parametrization, as well as the register of manufacturing orders and the analysis of historical data based on a real case.
\end{abstract}

Keywords: information sistems, manufacturing execution systems, MES

\section{Resumen}

En este trabajo se presenta la experiencia en el uso de un sistema de ejecución de fabricación, denominado MESView, para las sesiones prácticas de la asignatura Sistemas de Información del Máster Universitario de Ingeniería de Organización y Logística de la UPV. A través de un ejemplo sencillo basado en una empresa del sector de la industria alimentaria se presentan las utilidades del sistema de apoyo a la ejecución de fabricación seleccionado, su parametrización, así como el proceso de alta de órdenes de fabricación y análisis de datos históricos en un ejemplo basado en un caso real.

Palabras clave: sistemas de información, sistemas de ejecución de fabricación, MES 


\section{Introducción}

En el entorno de negocios actual, altamente globalizado y competitivo, caracterizado por una demanda cambiante y unos ciclos de vida de productos cada vez más breves se hacen necesarios profesionales capaces de eliminar las ineficiencias en los sistemas de producción con el objetivo de mejorar las operaciones logísticas que posibiliten el aumento de la productividad. En los próximos años, el sector industrial va a demandar un alto número de profesionales cualificados, con formación en las áreas de ciencia, tecnología, ingeniería y matemáticas (STEM, o Science, Technology, Engineering and Mathematics) (Randstad, 2016a, 2016b). Una de las titulaciones oficiales dentro del sistema universitario español relacionadas con estas áreas de conocimiento es el Grado en Ingeniería de Organización Industrial. En este ámbito, destacan diferentes artículos docentes que describen las competencias a adquirir por parte de los alumnos egresados, así como los diferentes perfiles de alumnos de ingreso (Marín García, García-Sabater, \& Canós-Darós, 2010; Marín-Garía, García-Sabater, Miralles, Villalobos, \& Romano, 2008). Asimismo, (Mula, DíazMadroñero, \& Poler, 2012) presentan las principales competencias genéricas, transversales y específicas de dicho título, así como una análisis de la estructura de los planes de estudio del Grado en Ingeniería de Organización en las diferentes universidades españolas.

En este ámbito, la Universitat Politècnica de Valéncia (UPV) ofrece un título de Grado en Ingeniería de Organización y un título de Máster Universitario en Ingeniería de Organización Logística (MUIOL). El objetivo de este Máster es formar profesionales para la empresa que sean capaces de analizar, modelar, diseñar, implantar y mejorar sistemas complejos con el fin de ofrecer productos y servicios en el menor plazo y con la mayor productividad, calidad, fiabilidad y eficiencia posible. Para ello, dicho título establece como una de las competencias específicas a adquirir por su egresados la habilidad de elegir y poner en funcionamiento los sistemas de información y las tecnologías de información y comunicaciones más adecuados que sirvan de apoyo al flujo de información a través de diferentes áreas de la empresa como compras, logística, producción, procesos, costes, recursos humanos, etc. En este contexto de negocio y control, los sistemas de ejecución de fabricación (en inglés, manufacturing execution systems o MES) permiten la optimización de las actividades de producción desde el lanzamiento de pedidos hasta los productos finales (MESA International, 1997a).

El objetivo de este trabajo es presentar la experiencia en el uso de un sistema MES en la asignatura de Sistemas de Información del MUIOL de la UPV. Mediante el uso de un sistema MES, a través de un ejemplo sencillo, se presentan al alumno las principales funcionalidades de la aplicación MESView relacionadas con un contexto de fabricación y control de producción. La contribución principal de esta comunicación es presentar el ejemplo empleado en la docencia de la citada asignatura, de forma que pueda servir de referencia como punto de partida para la elaboración de materiales docentes en el ámbito de las asignaturas relacionadas con los sistemas de información.

Este trabajo se organiza como sigue. En primer lugar, en el apartado 2 se describen las características principales de los sistemas MES. La sección 3 presenta la herramienta de

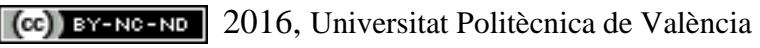

Congreso IN-RED (2016) 
software MESView. La sección 4 muestra un ejemplo ilustrativo de su aplicación. Finalmente, en la sección 5, se presentan las conclusiones de este trabajo.

\section{Sistemas MES}

En las últimas décadas, puede destacarse la inversión en sistemas de información por parte de multinacionales, grandes empresas, y últimamente cada vez más pequeñas y medianas empresas, con el objetivo de adquirir herramientas que permitan un incremento de su productividad. Este hecho puede constatarse en el desarrollo creciente del mercado de los sistemas de planificación de recursos de la empresa (ERP) en los últimos años. Sin embargo, a pesar de la presencia extendida de este tipo de sistemas, los ERP suelen no prestar atención al entorno de producción o taller (Holst 2011). Como consecuencia, muchas empresas optaron por desarrollar aplicaciones propias, adquirir otras específicas o utilizar hojas de cálculo o bases de datos para el apoyo a las operaciones de fabricación tales como la adquisición y monitorización de datos en tiempo real. Este tipo de aplicaciones software utilizadas para el control de procesos de producción, el aseguramiento de la calidad o el control de personal, generalmente, trabajaban de modo independiente sin ningún tipo de integración con el resto de sistemas de información. Sin embargo, en un entorno de producción real, estas tareas no pueden tratarse de modo aislado, debido a su interdependencia. En este contexto, se hizo necesaria la aparición de sistemas de información, los sistemas MES, que pudieran realizar una gestión y adquisición común de datos de las diferentes áreas del entorno de fabricación con el objetivo de asegurar un tiempo reducido de respuesta, la calidad, la reducción de costes y tiempos de producción.

Existen múltiples definiciones acerca de los sistemas MES. Entre ellas, el Bridgefield Group (2016) los define del siguiente modo:

"Un sistema de programación de la producción y seguimiento utilizado para analizar e informar de la disponibilidad de los recursos y su estado, programación y actualización de pedidos, recolección de datos detallados de ejecución tales como el uso de materiales, el uso de mano de obra, los parámetros del proceso, el estado del equipamiento y otra información crítica..."

En este sentido, con el objetivo de satisfacer las necesidades en entornos de fabricación diversos, (MESA International, 1997b) identificó las 11 funciones principales asociadas a los sistemas MES:

- Asignación y estado de recursos

- Programación detallada de operaciones

- Despacho/ejecución de unidades de producción

- Control de documentos

- Adquisición/recolección de datos

- Gestión laboral

- Gestión de la calidad

- Gestión de proceso

(c)) EY-NC-ND 2016, Universitat Politècnica de València

Congreso In-Red (2016) 
- Gestión de mantenimiento

- Seguimiento de productos

- Análisis de rendimiento

La publicación del estándar S95 por parte de Instrumentation, Systems \& Automation (ISA) Committee supuso la identificación de las interacciones entre las diferentes partes de los sistemas MES, así como la relación de éstos con el resto de sistemas de información de la empresa. Según el estándar S95, los sistemas MES se encuentran en una zona central, entre el nivel de fabricación donde se encuentran los sistemas automáticos de la línea de producción, y el nivel de negocio en el que se encuentran los sistemas de planificación logística, inventarios o sistemas ERP. El S95 está ampliamente extendido y facilitó a los proveedores de software empresarial una terminología y un modelo del flujo de información entre las diferentes áreas del entorno de fabricación y los diferentes departamentos de la empresa (Saenz de Ugarte, Artiba, \& Pellerin, 2009).

Las diferentes funcionalidades de los sistemas MES permiten mejorar el nivel de rendimiento global de la empresa y una mejor adaptación a los pedidos de los clientes. En este sentido, MESA International (1997a) indentificó de forma cuantitativa las mejoras en una empresa industrial representativa al utilizar un sistema MES: reducción del tiempo de ciclo $(45 \%)$, reducción del tiempo de procesado de datos de entrada entre sistemas independientes $(75 \%)$, reducción de la pérdida de información en papel $(57 \%)$, reducción de tiempo de fabricación (32\%), entre otras.

\section{MESView}

El desarrollo de MESView tiene sus orígenes en la fábrica de Ultracongelados de la Ribera (grupo ARDO) en Benimodo (Valencia). La fábrica cuenta con un departamento de TPM (Total Productivity Management) con una dilatada experiencia en proyectos de mejora contínua, reconocida con premios internacionales, que ha proporcionado las especificaciones funcionales de MESView.

MESView está orientada a optimizar el consumo de recursos y simplificar la gestión de los procesos.

En primer lugar, la herramienta proporciona control sobre el consumo de diferentes tipos de recursos: maquinaria, mano de obra, consumo de energía y materias primas. El control sobre el consumo de recursos se basa en los siguientes puntos:

- Monitorizar en tiempo real el consumo de recursos a través de un sistema de captura de datos.

- Calcular indicadores claves (KPIs) representativos del consumo de recursos.

- Comparar los indicadores obtenidos con un objetivo previamente fijado.

- Mostrar la información (indicadores y desviaciones con objetivos) en tiempo real e históricos a todo el personal involucrado en el proceso.

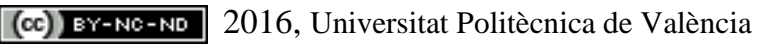

Congreso IN-RED (2016) 
En base a estos puntos, MESView integra diferentes aplicaciones que proporcionan soporte al desarrollo de proyectos de mejora continua. Desde la herramienta de configuración se pueden fijar los objetivos para los indicadores en una determinada iteración en el ciclo de mejora continua. Después, durante la ejecución, la herramienta muestra en tiempo real las desviaciones con respecto a los objetivos fijados, de manera que se puedan tomar medidas con carácter inmediato para minimizarlas. Por último, MESView proporciona herramientas para analizar los resultados, replanificar y revisar los objetivos para preparar una nueva iteración. Con esta metodología se pueden realizar proyectos de mejora sobre el consumo de cualquier tipo de recurso.

Por otro lado, MESView proporciona un soporte digital para automatizar los flujos de información con el plano operativo a nivel de planta y eliminar trámites en papel (controles de calidad, incidencias, hojas de ruta para el proceso). Al informatizar estos procesos, el intercambio de información es mucho más rápido (en tiempo real), seguro y fiable.

Una de las principales ventajas de MESView es su ergonomía. Es una herramienta visual y de fácil manejo, diseñada para que toda la organización pueda entender y analizar la información rápidamente. La aplicación es económica y rentable. El sistema de captura de datos utiliza la tecnología OPC UA (Open Platforms Communications Unified Architecture) que proporciona protocolos de comunicación que permiten conectarse virtualmente a cualquier dispositivo de control industrial. Esto permite aprovechar al máximo la infraestructura disponible en planta y minimizar los costes de implantación. Dada la amplitud de proyectos de mejora a los que puede dar soporte, el ahorro potencial es muy alto y el retorno de las inversiones muy rápido. Por otro lado, la aplicación no requiere de mucha infraestructura y la integración con otros sistemas de datos (p.ej. ERP) es sencilla. MESView se ha diseñado para permitir el cálculo de los indicadores a partir de un mínimo de variables industriales. A estas se pueden añadir nuevas variables para mejorar la capacidad de análisis que ofrece la herramienta, de manera que la instalación escale conforme a las necesidades de los proyectos de mejora. Por último, la aplicación es $100 \%$ web, por lo que se puede utilizar cualquier dispositivo como terminal como cliente.

MESView es una aplicación modular, de manera que el alcance de la aplicación se puede adaptar a las necesidades de cada instalación. Cada módulo está especializado en un área de aplicación de la mejora contínua. Los módulos son Eficiencia - control sobre la OEE y los tiempos de paro, Productividad - Control sobre la mano de obra, Mermas - Control sobre las materias primas y Energía - Control sobre la eficiencia energética.

MESView Eficiencia utiliza una variable industrial para determinar los tiempos de producción y paro de máquina. A través de la interfaz de usuario, el operario puede introducir la causa de paro seleccionando un estado de un árbol configurable por el usuario. Las causas de paro también se pueden detectar automáticamente por la aplicación a partir de señales de máquina. Los tipos de estado de paro contemplados por la herramienta son fallo, paro rutinario o no planificado. La aplicación también soporta el control de procesos manuales. En este caso, los tiempos de producción y paro se obtienen directamente de los estados introducidos manualmente por los operarios en la interfaz, sin necesidad de utilizar

(c)) EY-NC-ND 2016, Universitat Politècnica de València

Congreso In-Red (2016) 
ninguna señal de máquina. A partir de los tiempos de paro, la aplicación calcula la disponibilidad operacional como la ratio entre el tiempo de producción y el tiempo operacional (suma del tiempo de producción, el tiempo de paro rutinario y el tiempo de fallo) y la disponibilidad productiva como la ratio entre el tiempo de producción y el tiempo productivo (suma del tiempo de producción y el tiempo de fallo). Por otro lado, la aplicación controla la producción y las no conformidades de calidad. La aplicación soporta diferentes métodos para la captura de datos de producción: por muestreo de una variable industrial, por evento (por ejemplo, a través de una báscula), manual a través de la interfaz de usuario o por intercambio de datos con otros sistemas (por ejemplo, una aplicación de gestión de almacenes o el ERP de fábrica). A partir de los datos de producción y no conformidades, la aplicación calcula los indicadores rendimiento (ratio entre la velocidad real y la velocidad teórica) y calidad (ratio entre la producción conforme y la producción total). El indicador OEE (Overall Equipment Efficiency) se calcula como el producto de la disponibilidad operacional, el rendimiento y la calidad. Por otro lado, el indicador TEEP (Total Effective Equipment Performance) se calcula como el producto entre la disponibilidad productiva, el rendimiento y la calidad. La aplicación permite seleccionar uno de estos dos indicadores como el indicador de referencia para controlar la eficiencia. Por otro lado, la aplicación permite definir un objetivo para los estados de paro rutinario (por ejemplo, 30 minutos para un cambio de producto) y calcular el tiempo de exceso entre el tiempo de paro rutinario real y el objetivo. La aplicación también permite trabajar con un indicador de eficiencia que tiene en cuenta los tiempos de fallo y los tiempos de exceso como un indicador alternativo en el que únicamente se penaliza la eficiencia en los paros rutinarios cuando se excede el tiempo objetivo fijado para cada estado. Por último, para cualificar la pérdida de velocidad, la aplicación permite definir un umbral de velocidad lenta por producto. Si la velocidad baja de dicho umbral, la aplicación detecta el estado como estado de velocidad lenta. Por otro lado, la aplicación permite configurar diferentes señales de paro para detectar microparadas.

MESView productividad calcula la productividad de la mano de obra como la ratio entre las horas persona de los operarios asignados a un centro de coste y la producción realizada por el mismo. La aplicación proporciona asistentes para configurar las asignaciones de operarios a centros. Estas se pueden ajustar desde la interfaz de operario para aplicar correcciones sobre la asignación. Cada operario tiene asignado una categoría por defecto y una intensidad (por ejemplo, 50\% para un operario que está dando servicio a dos centros simultáneamente). Por otro lado, la aplicación permite definir un árbol de estados para el estado de los operarios (ausente, en línea). Con esto, MESView productividad permite calcular en tiempo real la productividad de la mano de obra directa ligada a producción, las desviaciones de categorías y el estado de los operarios.

Para cada producto, MESView permite definir una receta que define el consumo teórico de ingredientes para producir una unidad de producto final. A partir de variables industriales, MESView Mermas controla el consumo real de ingredientes y muestra calcula en tiempo real la merma real de cada ingrediente y la desviación con la merma objetivo definida por el usuario.

(c)) BY-NC-ND 2016, Universitat Politècnica de València 
Por último, MESView Energía proporciona control sobre el consumo de recursos energéticos en dos sentidos: control sobre el árbol de distribución y control sobre la eficiencia de actividades o usos energéticos. Para proporcionar control sobre el árbol de distribución energética, MESView muestrea el consumo reportado por medidores energéticos instalados en el árbol de distribución. MESView permite configurar nodos virtuales a partir de operaciones aritméticas (sumas y restas) sobre los contadores, de manera que se puede tener una estimación del consumo en áreas de la instalación donde no hay contadores físicos. Esto permite que el despliegue de los contadores sea gradual y la instalación se vaya adaptando a las necesidades de los proyectos de mejora de eficiencia energética. Por otro lado, MESView permite definir actividades energéticas de tres tipos: no productivas, productivas y de transformación. Cada una de estas actividades tendrá un tipo diferente de indicador de rendimiento energético (Energy Performance Indicator EnPI). Las actividades no productivas se caracterizan por una expresión de consumo, que son expresiones matemáticas que utilizan el consumo en nodos del árbol de distribución como variables, para el cálculo en tiempo real del EnPI (por ejemplo, kwh/m2). Las actividades productivas utilizan el cociente entre una expresión de consumo y la la producción en centros de coste para calcular en tiempo real EnPIs que tienen en cuenta el rendmiento energético por unidad producida (por ejemplo $\mathrm{kwh} / \mathrm{kg}$ ). Por último, las actividades de transformación se caracterizan por una expresión de consumo de un recurso energético y una expresión de producción de otro recurso. A partir del cociente entre estas dos expresiones, la aplicación permite controlar el rendimiento de actividades de transformación de un recurso energético a otro (por ejemplo, el rendimiento de una batería de compresores).

\section{Ejemplo de aplicación de MESView en la laboratorio}

La asignatura Sistemas de Información impartida en el MUIOL de la UPV corresponde con una asignatura de tipo obligatorio estructurada en créditos ECTS. Entre los principales contenidos de la misma destacan la aplicación estratégica de los sistemas de información (SI) y en las organizaciones, pero también un amplio recorrido por los aspectos básicos de los SI y una revisión de las aplicaciones esenciales en el entorno empresarial-industrial: como los sistemas de planificación de recursos de la empresa (ERP) y los sistemas para la ejecución y control de la producción (MES), entre otros. Asimismo, estos contenidos se consolidan mediante 15 horas de prácticas de laboratorio, de las cuales 4 horas, en sesiones de dos horas cada una, han sido programadas para el desarrollo de la presente experiencia relacionada con los sistemas MES.

La experiencia práctica que se presenta ha sido diseñada para ser realizada en grupos, preferentemente de 2 alumnos, con el objetivo complementario de favorecer la discusión y el análisis crítico. Como condición previa el alumnado debe conocer el funcionamiento básico de la aplicación MESview. Las tareas principales a realizar son 3:

1. Parametrización de un caso industrial.

2. Introducción y seguimiento de una orden. 
3. Análisis de los datos históricos de un periodo.

Se recomienda emplear las dos primeras horas para la tarea de parametrización, y dejar las dos horas de la siguiente sesión para las dos últimas tareas.

La tarea de parametrización consiste en el estudio de una empresa de envasado de verdura, aunque con datos alterados, e identificación de los valores correspondientes a las parámetros para el establecimiento de la estructura de la planta industrial y sus recursos, así como de sus características de funcionamiento en la aplicación MESView ${ }^{\circledR}$ para las condiciones de trabajo deseadas. En la Figura 1 se muestra la estructura de la planta, de la cual sólo se desea entrar en detalle del área de Multis y del recurso 2, que es una envasadora de ensaladillas.

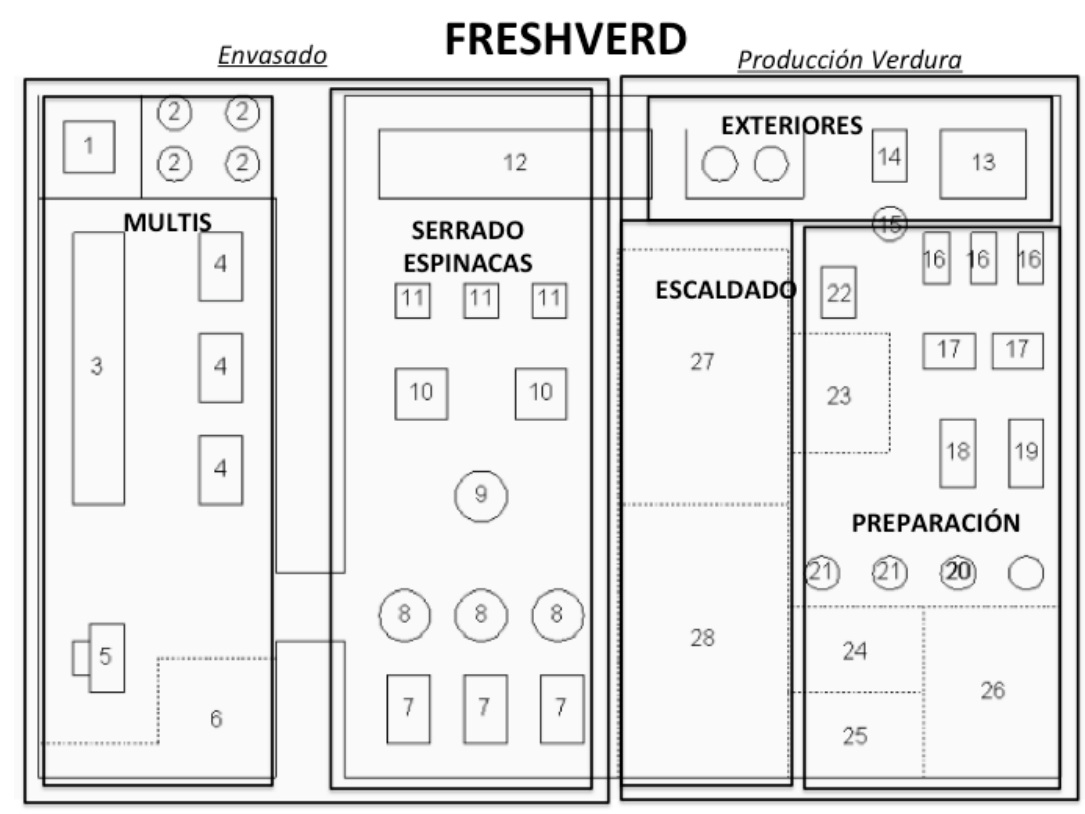

Fig. 1. Estructura de la planta de producción

Los alumnos reciben la información general sobre el funcionamiento del recurso 2. Se trata de un conjunto de máquinas que trabajan coordinadamente para el envasado de ensaladilla que después se congela, y que se envasa en cajas de diferentes tamaños, pero nunca de más de $1 \mathrm{Kg}$. El recurso está manejado por 2 operarios (fijo y eventual) con dedicación de un $100 \%$ a este recurso y un responsable de línea que trabaja al 50\% de dedicación. El recurso suele tener una perdida de unos 3 minutos por hora en pequeños paros no deseados, y además requiere de 6 minutos, cada hora, para realimentar las cajas del envasado. Las paradas de más 5 segundos son poco habituales. En este recurso se manejan 4 productos que son:

- EN10: Ensaladilla Normal de 205 gr.

- EN20 En saladilla Normal de 500 gr.

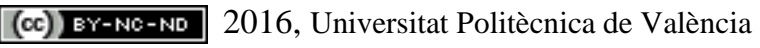


- EN10E Ensaladilla Extra de 250 gr.

- EN20E Ensaladilla Extra de 500 gr.

Para las cajas de 305 gr la velocidad máxima de envasado es de 1.600 cajas/hora, mientras que para las de 500 gr. es de 1.200 cajas/hora. Para los productos Normal (EN10 y EN20) se desea trabajar en condiciones de máximo rendimiento, ya que se entiende que el producto no es problemático. También se desea disponer de información relativa a la producción lenta de gran sensibilidad. Por otro lado, se sabe que la media de unidades/hora es en este caso de 1.450 uds para EN10 y de 1.050 uds. para EN20. Para los productos extras (EN10E y EN20E) se desea trabajar como mucho a un $80 \%$ de la velocidad máxima, ya que se entiende que el producto es más delicado. La información de producción lenta no debe ser muy sensible. También se sabe que la media de unidades/hora es en este caso de 1.200 uds. para EN10E y de 900 para EN20E.

Una vez los grupos han discutido las diversas alternativas y han definido la combinación más adecuada se realiza una puesta en común para conocer las opiniones de todos los grupos. Esta etapa suele durar aproximadamente entre 45-60 minutos. A partir de este momento se realiza una etapa más mecánica de introducción de los valores en la aplicación que suele durar unos 30 minutos, y una última etapa de verificación que suele durar unos 15 minutos. Esta primera sesión termina con un resumen de los hitos alcanzados y del sentido que tiene la práctica en el contexto de la asignatura.

La segunda sesión de la experiencia práctica suele realizarse una semana después de la primera. Este periodo sirve para que los alumnos revisen la sesión uno y maduren los conocimientos adquiridos ligados al cómo más que al para qué. Durante esta segunda sesión se abordará precisamente el para qué, y se realizarán las tareas 2 y 3.

Para la tarea 2, introducción y seguimiento de una orden, se pone en marcha un servidor de señales para el OPC asignado al recurso 2. De esta forma, se simula la existencia del recurso real, de forma que el responsable de la práctica puede mantenerlo en marcha o pararlo temporalmente simulando averías, paradas técnicas, etc.

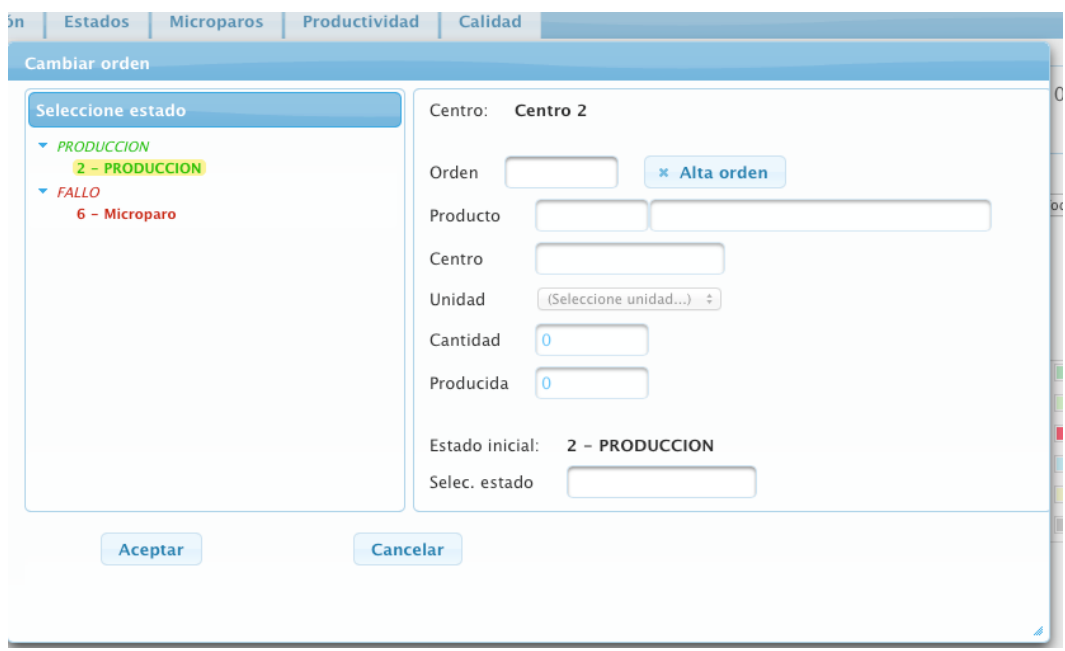

Fig. 2. Alta de órdenes de producción

(cc) EY-NC-ND 2016, Universitat Politècnica de València

Congreso In-Red (2016) 
Por su parte, cada grupo debe incluir una nueva orden de producción manual sobre el recurso 2 para alguna cantidad de unidades de alguno de los productos posibles (EN10, EN200, EN10E o EN20E) en el menú de monitorización, como se muestra en la Figura 2. Una vez, introducida la orden el grupo de alumnos puede realizar su seguimiento en el informe resumen. Estos datos son los mimos que puede ver en la tarea siguiente, la diferencia estriba en que en este caso la información varia en tiempo real en función de las velocidad la regularidad de la señal simulada. Esto permite que los usuarios aprecien los cambios de estado y vinculen la evolución de la señal recibida a los cambios en la información. Esta tarea se a revelado muy efectiva para que los usuarios comprendan la utilidad de la aplicación.

En la tarea 3, análisis de los datos históricos de un periodo, usando como base la pantalla que se muestra en la Figura 3. Con esta tarea se busca que el alumno entienda los conceptos de Velocidad Productiva, Eficiencia Productiva y Estados. Para ello se proporciona un número de orden cuyos datos son conocidos y cuyos cálculos de pueden reproducir fácilmente. Todos los conceptos son explicados combinado la información visual de la pantalla y el cálculo en la pizarra.

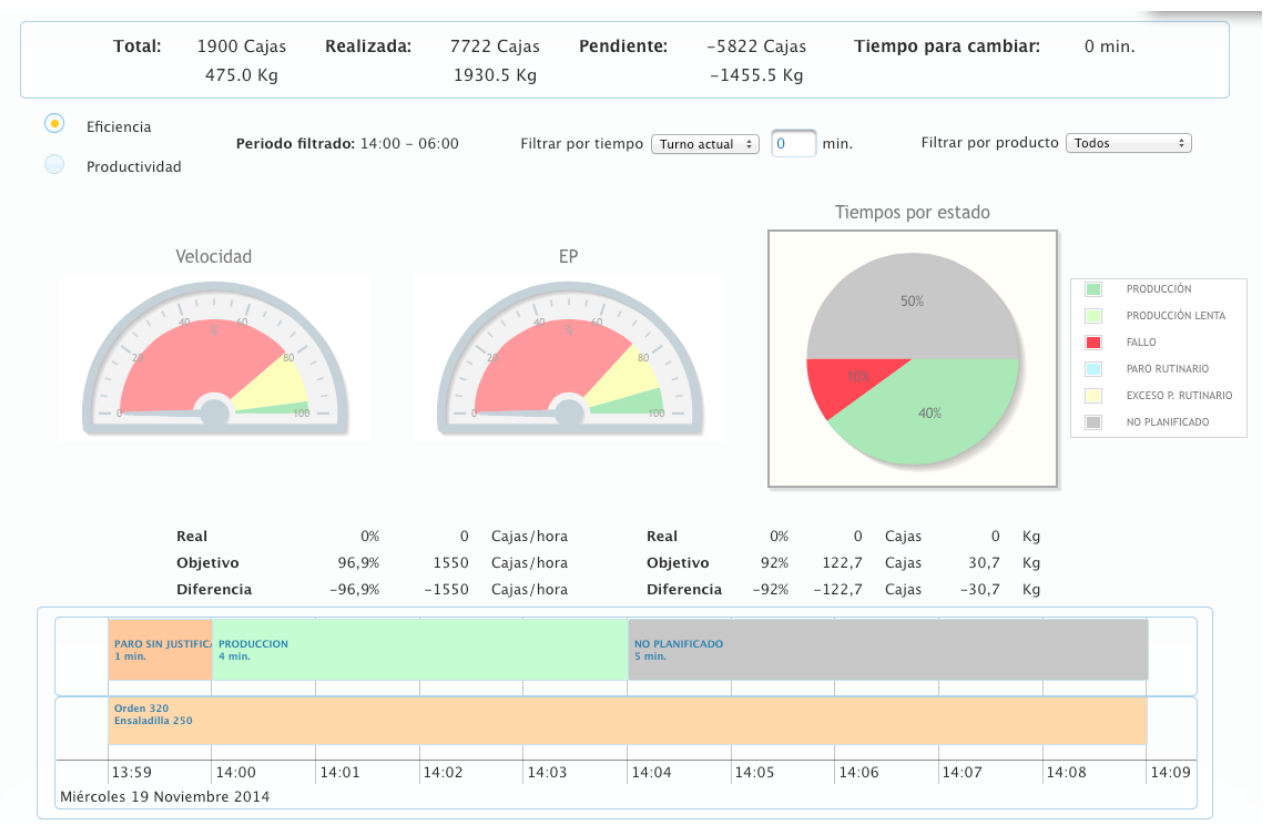

Fig. 3. Panel de análisis de datos (I)

La velocidad y la eficiencia productiva se muestran en un indicador gráfico y con un resumen numérico. En la zona inferior se muestra un diagrama de barras con la representación de la ocupación del centro y el estado de las diferentes órdenes. También se analizan el tiempo de trabajo y la productividad de cada orden, que también se muestran combinando datos numéricos y aspectos gráficos (Figura 4).

(cc)) EY-NC-ND 2016, Universitat Politècnica de València 


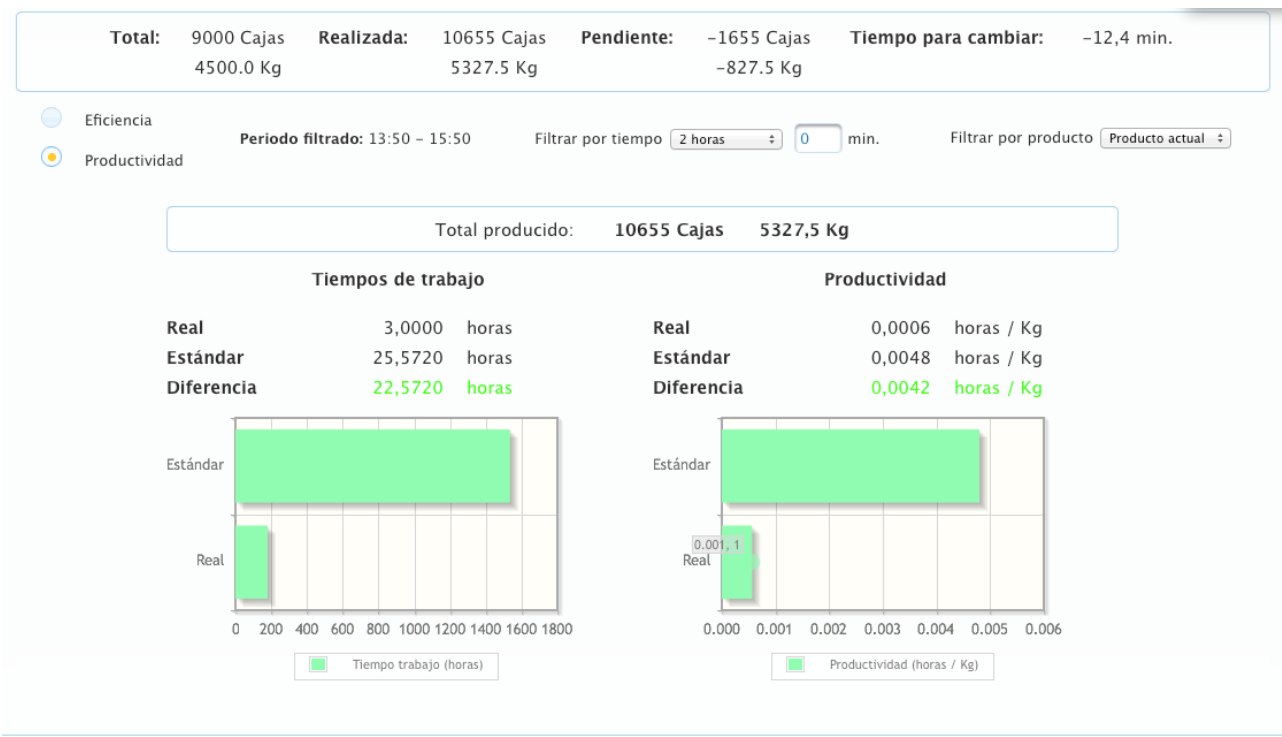

Fig. 4. Panel de análisis de datos (II)

Y finalmente en este ámbito de análisis se invita a los alumnos a realizar un estudio del conjunto de estados por el que ha pasado una orden (Figura 5) y cómo los tiempos asignados a cada estado contribuyen a la eficiencia y productividad previamente analizados.

\begin{tabular}{|c|c|c|c|c|c|c|c|c|c|c|}
\hline Resumen & Produ & cción & Estados & Microparos & Productividad & Calidad & & & & \\
\hline \multirow[t]{2}{*}{ Eliminar } & \multicolumn{10}{|c|}{ Nueva medida Exportar a XIS } \\
\hline & & & & roducto & & Fecha & Valor & Orden & Turno & Atributos \\
\hline 屯 & & EN20 - & Ensaladilla 500 & & & $/ 11 / 201415: 44$ & $\begin{array}{r}1776.0 \text { Cajas } \\
888.0 \mathrm{Kg}\end{array}$ & 120 & Resto & \\
\hline 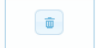 & & EN20 - & Ensaladilla 500 & & & /11/2014 15:29 & $\begin{array}{r}1776.0 \text { Cajas } \\
888.0 \mathrm{Kg}\end{array}$ & 120 & Resto & \\
\hline 苗 & & EN20 - & Ensaladilla $50 \mathrm{C}$ & & & /11/2014 15:14 & $\begin{array}{r}1776.0 \text { Cajas } \\
888.0 \mathrm{Kg}\end{array}$ & 120 & Resto & \\
\hline 元 & & EN20 - & Ensaladilla $50 \mathrm{C}$ & & & $/ 11 / 201414: 59$ & $\begin{array}{r}1775.0 \text { Cajas } \\
887.5 \mathrm{Kg}\end{array}$ & 120 & Resto & \\
\hline 画 & & EN20 - & Ensaladilla $50 \mathrm{C}$ & & & $/ 11 / 201414: 44$ & $\begin{array}{r}1776.0 \text { Cajas } \\
888.0 \mathrm{Kg}\end{array}$ & 120 & Resto & \\
\hline 画 & & EN20 - & Ensaladilla 500 & & & $/ 11 / 201414: 29$ & $\begin{array}{r}1776.0 \text { Cajas } \\
888.0 \mathrm{Kg}\end{array}$ & 120 & Resto & \\
\hline 画 & & EN10 - & Ensaladilla 250 & & & /11/2014 14:14 & $\begin{array}{r}1775.0 \text { Cajas } \\
443.75 \mathrm{Kg}\end{array}$ & 320 & Resto & \\
\hline
\end{tabular}

Fig. 5. Panel de análisis de datos (III)

Con estas tres tareas el alumno debe alcanzar una idea global sobre el empleo de las herramientas MES, comenzando con el diseño y terminando con el análisis de los datos.

En la Figura 6, se resume mediante un esquema las tareas a realizar en cada una de las dos sesiones, indicando la secuencia de las mismas y una duración orientativa.

(cc) EY-NC-ND 2016, Universitat Politècnica de València

Congreso In-Red (2016) 


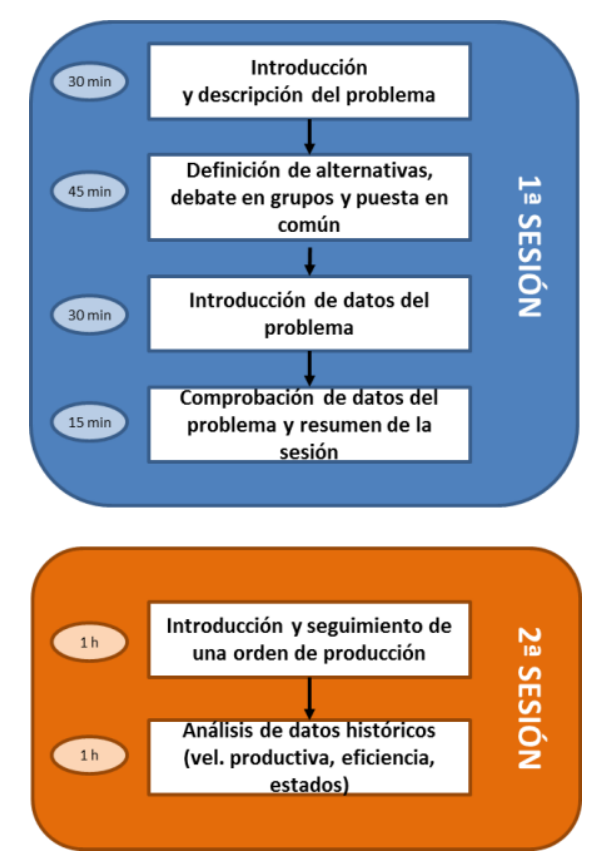

Fig. 6. Esquema resumen de la aplicación de MesView en laboratorio

\section{Conclusiones}

En este trabajo, se ha presentado la experiencia en el uso de un sistema MES para la docencia de la asignatura de Sistemas de Información en el MUIOL de la UPV. En primer lugar, se ha contextualizado la importancia de la docencia de este tipo de sistemas de información a los alumnos del área de ingeniería de organización. A continuación se han expuesto las principales características de los sistemas MES, incidiendo en las ventajas que pueden aportar y cómo se relacionan con el resto de sistemas de información existentes en en un empresa industrial. Posteriormente, se describen las funcionalidades del sistema MES seleccionado, denominado MESView. Por último, se detalla cómo se han programado las sesiones prácticas en el laboratorio así como el ejemplo utilizado como hilo conductor para el uso de MESView basado en un ejemplo real. A través de éste y los ejercicios propuestos, los alumnos pueden conocer el proceso de parametrización del sistema, alta de órdenes de fabricación y el análisis de datos históricos asociados a un periodo y a la planta de fabricación que se desea controlar con el sistema MES elegido.

\section{Referencias}

Bridgefield. (2016). Bridgefield Group Supply Chain Glossary. Retrieved April 5, 2016, from http://www.bridgefieldgroup.com/glossarym.html

Holst, L., (2001). Integrating discrete-event simulation into the manufacturing system development process. Lund University, Department of Mechanical Engineering, Division of Robotic

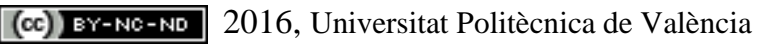

Congreso IN-RED (2016) 
MESA International (1997a). MES explained: a high level vision. MESA International White Paper 6 (Vol. 1).

MESA International (1997b). MESA White Paper \#02: MES Functionalities and MRP to MES Data Flow Possibilities. Retrieved from https://services.mesa.org/ResourceLibrary/ShowResource/2cedfe75-daed-4b9c-b187f421cf90fdd 2

Marín García, J. A., García-Sabater, J. P., \& Canós-Darós, L. (2010). La Ingeniería de Organización ante el diseño de los nuevos títulos europeos. Dirección $Y$ Organización, $\quad O(41), \quad 35-43 . \quad$ Retrieved from http://ww.revistadyo.com/index.php/dyo/article/view/336

Marín-Garía, J. A., García-Sabater, J. P., Miralles, C., Villalobos, R., \& Romano, C. A. (2008). La Ingeniería de Organización en un entorno educativo globalizado: reflexiones y propuestas para la universidad española. $\{I I\}$ International Conference on Industrial Engineering and Industrial Management, 817-826. Retrieved from http://adingor.es/congresos/web/articulo/detalle/a/285

Mula, J., Díaz-Madroñero, M., \& Poler, R. (2012). Configuración del Grado en Ingeniería de Organización Industrial en las universidades españolas. Dirección y Organización, O(47), 5-20. $\quad$ Retrieved from http://www.revistadyo.com/index.php/dyo/article/view/398

Randstad. (2016a). El sector industrial en España necesitará 3,5 millones de profesionales especializados en 2025. Retrieved from http://www.randstad.es/nosotros/salaprensa/el-sector-industrial-en-espana-necesitara-mas-de-tres-millones-deprofesionales-especializados-en-2025

Randstad. (2016b). Randstad workmonitor. Retrieved from http://www.randstad.es/tendencias360/video-randstad-workmonitor-2016

Saenz de Ugarte, B., Artiba, a., \& Pellerin, R. (2009). Manufacturing execution system - a literature review. Production Planning \& Control, 20(January 2015), 525-539. http://doi.org/10.1080/09537280902938613 